\title{
Wirksamer integrieren: Gesundheitsberufe als Erfolgsfaktor
}

\author{
Drohende Versorgungslücken, zum Beispiel bei der hausärztlichen Betreuung oder \\ der Behandlung chronisch kranker Patienten, rücken Themen wie Teammedizin, \\ Delegation und Substitution von Aufgaben ins Rampenlicht. Die Patienten erwarten \\ zu Recht, dass diese Themen zu ihrem Nutzen bearbeitet und umgesetzt werden - \\ ein Plädoyer für mehr interprofessionelle Teamarbeit statt interprofessionellem \\ Verschieben von Aufgaben.
}

Vorstand des fmc das Schweizer Forum für Integrierte Versorgung
Korrespondenz:

PD Dr. Peter Berchtold

Freiburgstrasse 41

CH-3010 Bern

peter.berchtold[at]college-m.ch
Niemand in der Gesundheitsversorgung, unabhängig vom fachlichen Hintergrund, würde infrage stellen, dass Interprofessionalität, verstanden als Zusammenarbeit zwischen ärztlichen und nicht-ärztlichen Gesundheitsberufen, sowie interprofessionelle Teamarbeit zu den Schlüsselfaktoren für die Patientenbetreuung der Zukunft gehört. Gleichzeitig zeigen die aktuellen Diskussionen zur parlamentarischen Initiative «Gesetzliche Anerkennung der Verantwortung der Pflege», wie schnell sich die Professionen im Gestrüpp von Missverständnissen und politisch motivierten Stellungnahmen verheddern.

Interprofessionalität und Teamarbeit sind zwei unabdingbare Ingredienzen der Integrierten Versorgung. Das diesjährige Symposium des fmc Forum Managed Care stellt sie deshalb ins Zentrum (siehe Kasten nächste Seite). Wir haben vier ausgewiesene Experten aus unterschiedlichen Professionen und Bereichen eingeladen, zu drei Thesen aus diesem Themenkreis Stellung zu nehmen. Die Experten sind:

- Prof. Dr. Jürg H. Beer, Chefarzt Medizinische Klinik, Kantonsspital Baden;

- Claudia Galli, Präsidentin Schweizerischer Verband der Berufsorganisationen im Gesundheitswesen SVBG;

- Prof. Dr. Rebecca Spirig, Direktorin Pflege und MTTB Universitätsspital Zürich und Professorin Institut für Pflegewissenschaft Universität Basel;

- Dr Brigitte Zirbs Savigny, FMH médecine int. gén., Membre comité de MFE.

Die Antworten unterstreichen vor allem eines: Die genannten Themen stehen überall zuoberst auf der Prioritätenliste. Die Stellungnahmen zeigen aber auch wesentliche Unterschiede, ohne deren Klärung keine wirkliche interprofessionelle Teamarbeit möglich wird.

These 1: Mehr Interdisziplinarität meint auch mehr Interinstitutionalität. Es gilt daher in erster Linie die Vernetzung zwischen Leistungserbringer-Organisa- tionen (z. B. Ärztenetzen, Spitälern, Rehakliniken, Apotheken, Spitex, Heime) zu intensivieren!

Jürg Beer: Bisher hatte der Patient weitgehend allein den «Marsch durch die Institutionen» zu bewältigen. Künftig wollen Patienten mit System und Empathie begleitet werden. Die Interinstitutionalität kann und soll deshalb gefördert und gefordert werden, durch die Kantone (z. B. Projektunterstützung), durch die Versicherer (z. B. Komplexpauschalen, Übernahme von koordinierenden Home Visits, Kostengutsprache innerhalb 24 Stunden), durch die Spitäler (z. B. intelligentes Care Management). Es gibt bereits viele hervorragende Projekte, die funktionieren, aber leider nicht bekannt sind. Empfehlung: Eine elektronisch zugängliche Projektlandschaft soll durch eine unabhängige Organisation (z. B. fmc!) koordiniert und bewertet werden.

\section{«Bisher hatte der Patient weit- gehend allein den «Marsch durch die Institutionen> zu bewältigen.»}

(Jürg Beer)

Rebecca Spirig: Die komplexen und vermehrt chronischlangzeitlichen Krankheiten von Patienten erfordern zunehmend eine auf die spezifische Situation abgestimmte Behandlung durch Expertinnen und Experten aus unterschiedlichen Feldern. Es gilt, diese Einzelleistungen z.B. durch Hausärzte, Fachärzte, die Spitäler, Physiotherapeuten und Pflegefachpersonen aus der Spitex auf die Behandlungspfade der Patienten auszurichten. Die optimale Vernetzung zwischen Leistungserbringer-Organisationen zum Wohle der Patienten und für verbesserte Patientenergebnisse ist deshalb dringend notwendig. Internationale und nationale Erfahrungen zeigen zudem, wie wirksam innovative Vernetzungen und Zusammenarbeitsformen sind. 


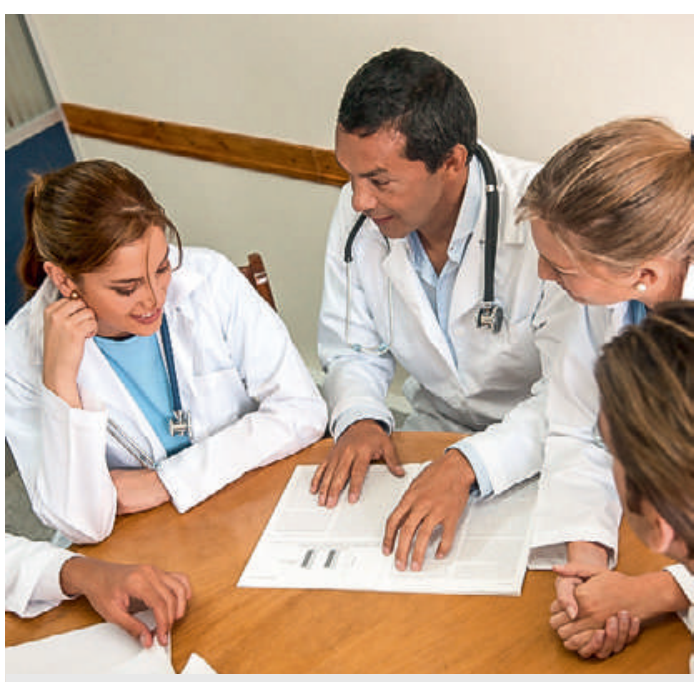

Durch interprofessionelle Zusammenarbeit wird das Potential der Gesundheitsberufe zugunsten der Patienten optimal genutzt.

Claudia Galli: Übergangssituationen zwischen den Institutionen brauchen sorgfältige Vorbereitung und Begleitung. Drehtür-Effekte sollen verhindert und erfolgreiche Reintegration in den (Arbeits-) Alltag unterstützt werden. Eine gezielte Schnittstellenpflege stellt sicher, dass die Betreuung der betroffenen Personen nahtlos weitergeht und relevante Informationen für die zuständigen Fachpersonen zugänglich sind. E-Health spielt dabei eine wichtige Rolle: Zugang zu Patientenund Behandlungsdaten und zu Verlaufsinformationen bilden eine wichtige Stütze in diesen Prozessen. Die Vernetzung zwischen den Institutionen bildet dazu sicherlich eine gute Voraussetzung; umgesetzt werden muss sie aber primär zwischen einzelnen Fachpersonen, die interprofessionell zusammenarbeiten.

Brigitte Zirbs Savigny: Interdisciplinaire n'est pas synonyme d'interprofessionnel. L'interdisciplinarité définit la collaboration entre médecins qualifiés de formations ou spécialités différentes autour d'un patient avec une pathologie définie - exemple: les tumor-boards. L'interprofessionnalité est un système plus complexe qui réunit autour d'un patient plusieurs professionnels des soins, avec des niveaux de formations et de compétences différentes (infirmière, Spitex, pharmacien, ergothérapeute, physiothérapeute, médecin de famille, assistante sociale) qui assurent ensemble un suivi sur le long terme. Dans ce contexte la communication et l'échange d'informations sont les éléments clés de la qualité. On parle de leadership situationnel: la responsabilité est assurée par le professionnel qui dispose des compétences les plus pointues en fonction du problème abordé.

These 2: Mehr Interdisziplinarität meint auch mehr Interprofessionalität. Wichtige Voraussetzung dafür sind neue Aufgabenverteilungen zwischen den Gesundheitsberufen, insbesondere zwischen ärztlichen und nicht-ärztlichen Berufen.
Jürg Beer: Interprofessionalität ist heute Chance und morgen Notwendigkeit. Der Druck des Ärztemangels wird die praktische Umsetzung neuer Aufgabenverteilungen und Zusammenarbeitsformen zwischen den Gesundheitsberufen massiv beschleunigen. Die Integration von Berufsgruppen wie Medizinische Praxisassistentinnen, die bereits ausgezeichnete Stützen sind in der ärztlichen Praxis, ist bereits eine Erfolgsgeschichte im Spital; Nurse Care Managerinnen (NCM) erleichtern die Abläufe der Abteilungsärzte im Spital und integrierte Nurse Care Practitioners (NCP) ergeben im Team mit der Ärzteschaft ein interessantes Arbeitsmodell.

\section{«Für die Behandlung durch die verschiedenen ärztlichen und nicht- ärztlichen Berufsangehörigen sind klare Zuständigkeiten zu formulieren.» (Rebecca Spirig)}

Rebecca Spirig: Interdisziplinarität allein reicht heutzutage nicht mehr aus, denn insbesondere Patienten mit chronisch-langzeitlichen Krankheiten sind auf eine gut funktionierende und abgestimmte Interprofessionalität angewiesen. Für die Behandlung durch die verschiedenen ärztlichen und nicht-ärztlichen Berufsangehörigen sind klare Zuständigkeiten zu formulieren. Patienten müssen wissen, wer ihnen welche Behandlung anbietet und wer für welche Aufgabe vorbereitet ist. In vielen Feldern gilt es die Aufgabenverteilungen neu zu definieren, denn internationale Erfahrungen und Studien zeigen deutlich, dass nichtärztliche Berufsangehörige mehr Aufgaben übernehmen können als in der Schweiz bisher für sie vorgesehen sind - mit positiven Ergebnissen für die Patienten.

Claudia Galli: Interprofessionalität ist zentral für die zukünftige Gesundheitsversorgung. Dabei werden nicht einfach Aufgaben von einem Beruf zum anderen verschoben. Neue Aufgabenteilungen bringen Fragen zu Kompetenzen, Verantwortung und Haftung hervor. Dabei wird auch die Grenze zwischen Gesundheits- und sozialen Berufen durchlässiger. Wenn ernsthaft interprofessionell zusammengearbeitet wird, kann das Potential der Gesundheitsberufe zugunsten der Patienten ausgeschöpft werden. Am Beginn einer solchen Entwicklung steht etwas ganz Einfaches: Die Gesundheitsberufe müssen mehr voneinander wissen. Und sie müssen die Bereitschaft mitbringen, eingefahrene Wege zu verlassen. Initiativen zur Stärkung der interprofessionellen Aus- und Weiterbildung zeigen hier in die richtige Richtung. 
Brigitte Zirbs Savigny: Les médecins ont parfois de la peine à lâcher prise au sein d'un modèle qui donne la responsabilité d'un problème à une infirmière, par exemple. Peur de perdre le prestige, peur de perdre du

\section{«Nous devons encore apprendre à avoir confiance sans commander!» \\ (Brigitte Zirbs Savigny)}

\begin{abstract}
pouvoir? Nous devons encore apprendre à avoir confiance sans commander! Répartir les tâches selon un modèle interprofessionnel dans le cadre d'une équipe interdisciplinaire signifie que chacun accepte de lâcher prise sur son pouvoir en faveur d'un dialogue constructif dont l'aboutissement unique et original est le bien du patient et la qualité des soins qui lui seront prodigués. Sera promu responsable situationnel celui qui est le plus compétent dans le cadre d'un problème précis et pas forcément celui qui coordonne la globalité de la prise en charge, à savoir le médecin de famille.
\end{abstract}

These 3: Mehr Interprofessionalität ist vor allem im ambulanten Bereich notwendig, um die hausärztliche Versorgung zu ergänzen und zu entlasten. Voraussetzung dafür ist, dass Pflegefachpersonen Leistungen in eigener fachlicher Verantwortung also ohne ärztliche Verordnung - erbringen dürfen.

Jürg Beer: Obwohl dies in vielen Ländern bereits Realität und in gewissen medizinischen Disziplinen Routine ist (z. B. Geburtshilfe, Wundpflege, Palliative Care, Peritonealdialyse), sind die Gräben und Differenzen noch gross. Deshalb ist ein behutsames, schrittweises Vorgehen angezeigt, vorerst wohl ohne vollständige eigene Verantwortung und unter ärztlicher Supervision im Sinne einer erweiterten Delegation. Gleich-

\section{fmc-Jahressymposium 2014 am 18. Juni im Hallenstadion Zürich}

Das Jahressymposium 2014 des fmc Forum Managed Care beleuchtet unter dem Titel «Wirksamer integrieren: Gesundheitsberufe als Erfolgsfaktor» die Möglichkeiten und Herausforderungen der interdisziplinären Betreuung: Welche Gesundheitsberufe - Pflegefachpersonen, Physiotherapeutinnen, Psychologen, Apotheker, Medizinische Praxisassistentinnen u. a. - können welche Rolle spielen? Was können Ärzte und Ärztinnen durch neue Aufgabenteilungen gewinnen? Wie lässt sich eine interprofessionelle Arbeitsteilung unter diesen Vorzeichen organisieren? Welche Erfahrungen und Erkenntnisse gibt es in der Schweiz und im Ausland mit interdisziplinären Betreuungsteams? Wie reagieren Patientinnen und Patienten auf diese neuen Modelle und welchen Nutzen haben sie davon? Solche und ähnliche Fragen werden in Referaten, Projektpräsentationen und Workshops diskutiert. Hinzu kommen die Vergabe des fmc-Förderpreises 2014 sowie das Networking Dinner. Das laufend aktualisierte Programm mit der Möglichkeit zur Anmeldung finden Sie unter www. fmc.ch/symposium.

Das fmc-Symposium 2014 wird vom Schweizerischen Institut für ärztliche Weiter- und Fortbildung (SIWF) mit 5 Credits anerkannt. zeitig stimmen mich meine Erfahrungen einer Kooperation von NCP, Haus- und Spitalärzten in New York im Sinne der These zuversichtlich. Hier bräuchte es mehr politische Akzeptanz und versicherungsseitig anerkannte Abrechnungsmöglichkeit und finanzierte Pilotprojekte mit überzeugenden Resultaten.

Rebecca Spirig: In der ambulanten Versorgung sind Innovationen dringend notwendig. Zu den nicht-ärztlichen Berufsangehörigen zählen heute diverse Berufsgruppen. Diese können insbesondere in der hausärztlichen Versorgung gezielt und für Patienten wirksam eingesetzt werden. Dies konnte in internationalen Studien insbesondere bei Tätigkeiten von diplomierten Pflegefachpersonen und Pflegeexpertinnen APN mehrfach nachgewiesen werden. Um diese sinnvoll resp. eigenverantwortlich einzusetzen, gilt es jedoch die fachliche Verantwortung der diplomierten Pflegefachpersonen und Pflegeexpertinnen APN neu zu regeln.

\section{«Die Pflege umfasst einen mit- verantwortlichen und einen eigen- verantwortlichen Bereich. »}

(Claudia Galli)

Claudia Galli: Unbestrittenermassen benötigt die hausärztliche Versorgung im ambulanten Sektor Entlastung und sinnvolle Ergänzung. Und Pflegefachpersonen sollen bestimmte Leistungen ohne ärztliche Verordnung erbringen können. Die Pflege umfasst einen mitverantwortlichen und einen eigenverantwortlichen Bereich. Heute schon erbringen Pflegefachpersonen Leistungen, deren ärztliche Verordnung eine rein administrative Pflichtübung ist, damit sie von der Krankenkasse bezahlt werden. Gemeint sind beispielsweise Leistungen der Grundpflege, Bedarfsabklärungen und pflegerische Beratung. Solche Leistungen sollen - unabhängig von der Frage der Entlastung der Hausärzte - ohne ärztliche Verordnung erbracht und abgerechnet werden können.

Brigitte Zirbs Savigny: L'interprofessionnalité n'est pas seulement indispensable pour décharger de certaines tâches le travail ambulatoire du médecin de famille, mais également pour faciliter la continuité des soins dans le cadre de maladies chroniques multi-morbides. Dans un contexte idéal, c'est une évidence de penser que celui qui porte la responsabilité de la tâche devrait aussi en porter la responsabilité financière. Le système tarifaire actuel ne prévoit que peu de prestations pour facturer le travail du personnel de soin, la coordination du suivi. Il paraît nécessaire d'envisager une révision du modèle tarifaire pour y inclure, entre autre, des prestations pour les professionnels de soin, facturables sous leur entière responsabilité. 